\title{
Physiognomische Prämissen des Castings in der Filmproduktion
}

"Some people come across as sad (a naturally turned down mouth); others as cheerful (a naturally upturned mouth); as sensuous (a wide wide mouth with pouting lips); as serious (thin lips) etc. ${ }^{1}$ So lautet die ebenso nüchterne wie ernüchternde Bilanz des britischen Regisseurs Patrick Tucker, der sich ebenso kritisch wie konstruktiv mit der weit verbreiteten, typologisch verfahrenden Besetzungspraxis in der Filmproduktion auseinandersetzt. Die Analyse der Besetzung von Filmfiguren, die Rekonstruktion von Entscheidungen im Verlauf von Castingprozessen und der Vergleich von verschiedenen Verkörperungen ein- und derselben Figur bei mehrfach verfilmten literarischen Stoffen bergen je für sich Potentiale, die, so meine These, eine besondere Affinität zum Komplex der Physiognomik aufweisen. Inwieweit, so wird zunächst zu fragen sein, führt das Filmcasting eine von Stereotypen geprägte Zuschreibungspraxis fort, die aus dem konkreten Erscheinungsbild eines Gesichtes nicht nur die jeweilige emotionale Gestimmtheit der Person ableitet (Pathognomik), sondern, allgemeiner, aus Merkmalen des Gesichts, der Gestalt und der Proportionen seiner Teile allgemeine Charaktereigenschaften der Person abstrahiert (Physiognomik)? Diese Praxis in Castingprozessen mit einem methodologisch gesicherten Verfahren zu untersuchen, erforderte eine aufgrund von teilnehmender Beobachtung und Interviews zu erstellende Diskursanalyse charakteristischer Modi der Entscheidungsfindung bei der Besetzung von Filmrollen, was nur im Rahmen einer längerfristigen Studie geleistet werden könnte. Mein Beitrag versteht sich als vorläufiger skizzenhafter Problemaufriss zu diesem Feld.

Vom gedrehten Material ausgehend, sollen zudem in einer kleinen Fallstudie die aus filmästhetischer Perspektive zu bedenkenden Faktoren anhand der Besetzung der Figur des Edward Rochester in den drei jüngsten Film-/ TV-Adaptionen von Charlotte Brontës Roman Fane Eyre diskutiert werden.

1 Patrick Tucker: Secrets of Screen Acting (1994), 2. Aufl., New York/London 2003, S. 84. 
Diese Gruppe von Adaptionen bietet sich auch insofern gut an, als in ihrem als autobiografische Ich-Erzählung ausgewiesenen Prätext physiognomisches -Wissen nicht nur als Orientierungswissen, auf das sich die Hauptfigur stützt, eine wesentliche Rolle spielt; es wird als soziale Praxis in der dargestellten Welt auch reflexiv und dadurch problematisiert. Das Schließen von der äußeren Gestalt und den Gesichtszügen einer Figur auf deren Charakter ist in Fane Eyre in einen physiognomischen Diskurs eingebettet, der sich von der wertenden Verknüpfung - körperliche Schönheit geht einher mit einem guten Charakter, körperliche Hässlichkeit hingegen mit einem schlechten - entschieden löst. Der Roman gibt stattdessen einem sneuen Sehen v von Körperverhältnissen Raum und identifiziert dieses Sehen als ein spezifisch weibliches.

1. Die Arbeit der Besetzung: Vom sinneren Bild zum ıgefundenen Körperı

Castingprozesse setzen beim Drehbuch an. Je nachdem wie explizit rausgeschrieben eine Figur auch im Hinblick auf ihre äußere Erscheinung ist, lenkt der Text die Vorstellung der Filmschaffenden mehr oder weniger stark. Es bilden sich, wie bei jedem Leseakt, innere Vorstellungsbilder darüber aus, auf welche mögliche Körperlichkeit die in einem Satz von Merkmalen erfasste und durch ihr Handeln konturierte Figur referiert. Aus dem Genre, dem sich der zu drehende Film ferner zuordnet, resultieren weitere Anforderungen, welchen die für eine Besetzung in Frage kommenden Schauspielerinnen und Schauspieler genügen müssen. Die zunächst (und bis zum Vorsprechen) um eine Gruppe von Darstellern kreisenden Vorstellungen gilt es im Besetzungsprozess so weit einzugrenzen, bis die Person gefunden ist, die für die zu vergebende Rolle als richtige identifiziert wurde. Der so rgefundene Körper ist damit Ergebnis einer sich immer mehr verfeinernden Wahl aus möglichen Darstellern.

In der Filmkritik finden sich vereinzelt Überlegungen darüber, was gewesen wäre, wenn Schauspielerin oder Schauspieler X und nicht Y die Hauptfigur $\mathrm{Z}$ in einem kanonisierten Filmwerk gespielt hätte. ${ }^{2}$ Solche - letztlich müßigen - Spekulationen weisen der Besetzung zu Recht eine eminente Rolle zu,

2 Vgl. etwa Linda Rosenkrantz: The role that got away, in: Film Comment 14/1 (1978), S. 42-48. Gleichwohl ist es reizvoll, sich vorzustellen, wie Cary Grant anstelle des besetzten James Mason die männliche Hauptfigur Norman Maine in George Cukors A Star is Born (USA 1954) gespielt hätte. Vgl. die Liste in Rosenkrantz' Artikel, S. 48. 
denn nicht nur das gesamte körperliche Erscheinungsbild einer Darstellerin oder eines Darstellers ist im Film relevant, auch ihre oder seine Stimme und deren intonatorische Variationsbreite prägen die Gestaltung einer Figur. Bleiben wir zunächst bei der Stimme: Abgesehen von der Fähigkeit, Dialekte oder sprachliche Färbungen authentisch sprechen und nuancieren zu können, ist vor allem die Stimmhöhe einer Schauspielerin oder eines Schauspielers von Bedeutung für eine Besetzung. ${ }^{3}$ So äußerte sich, um ein aktuelles Beispiel zu nennen, der britische Schauspieler Richard Armitage, der über eine markante Sprech- und Singstimme in der Baritonlage verfügt, in einem Interview zu seiner Gestaltung der Figurenstimme von Thorin Oakenshield, dem exilierten, heroischen Thronanwärter des Zwergenreichs Erebor in Peter Jacksons Hobbit-Trilogie (NZ/GB/USA 2013-2015): »I just wanted it to sound like that kind of character that could command an army over a battlefield. It's a specific sound that I was looking for. ${ }^{4}$ Diese um zwei Halbtöne abgesenkte Stimmlage artifiziell herzustellen und sie durch die Trilogie hindurch zu tragen, bedeutete eine große Anstrengung.

Obwohl Stimme, Alter und Statur von Schauspielerinnen und Schauspielern zentrale besetzungsrelevante Faktoren sind, kommt dem Gesicht aufgrund der Häufung dramaturgisch exponierter Großaufnahmen in der visuellen Auflösung des Films eine elementare Rolle zu. Die Mimik, die seit der Aufklärung in Schauspieltheorien eine prominente Position einnimmt, ${ }^{5}$ ist für das Verständnis der Figurenemotionen, für das Entstehen von Sympathie, Antipathie oder Empathie im kommunikativen Spiel der Übertragung von Emotionen zwischen Figur und Zuschauer zentral. ${ }^{6}$ Im Film- und Fernsehschauspiel treten allerdings Faktoren hinzu, die traditionelle, für das Bühnenschauspiel entwickelte physiognomische bzw. pathognomische Deutungsmuster nicht problematisieren. Nicht nur die Maske, sondern vor allem auch die Lichtsetzung bilden neben Kameraoptik (Brennweiten) und Perspektivierung wirkungsvolle optionale Parameter der sich im Zeitfluss des Films

3 Vgl. zur Stimmlichkeit und zum Begriff einer 'Physiognomie der Hörwelt‘ Ursula von Keitz: Laut(er) Flächen. Stimme und Apparat im Frühen Tonfilm, in: Mehr als Schein. Oberflächenphänomene in Film, Kunst, Literatur und Theater, hg. von Hans-Georg von Arburg u.a., Zürich/Berlin 2008, S. 187-200.

4 Interview mit Richard Armitage (und Lee Pace) zu The Hobbit. The Battle of the five Armies (2014), zit. nach: www.youtube.com/watch?v=NdwCLzHMnIs (18. März 2016).

5 Vgl. Alexander Košenina: Anthropologie und Schauspielkunst. Studien zur »eloquentia corporis« im 18. Jahrhundert, Tübingen 1995.

6 Vgl. Philipp Brunner: Augenblicke des Gefühls. Gesichter in Großaufnahme, in: HansGeorg von Arburg u.a. (Hg.): Mehr als Schein, S. 201-217. 
stetig wandelnden cinematischen Kommunikation der Zuschauer mit dem Figuren- bzw. Schauspielergesicht. Sie beeinflussen die Interpretation von Charakteren entscheidend mit, was Jacques Aumont zu der provokativen Behauptung veranlasst hat, dass Großaufnahmen zwar nach wie vor dramaturgisch bedeutsam seien, sich aber weniger für »das dargestellte Gesicht als für die Darstellung selbst« interessieren würden. ${ }^{7}$ Aumont, dessen Befund sich auf einen historischen Wandel in der filmischen Gesichtsdarstellung stützt, verkennt meines Erachtens hier allerdings, dass das gefilmte Gesicht in der Einstellung nie für sich allein stand und steht, sondern immer im Kontext des kadrierten Bildraums gesehen wurde und wird. Das Licht definiert die Bedingungen seines Erscheinens, es gibt ihm seine Helligkeits- und Schattenwirkungen, die ihrerseits das mimische Spiel unterstreichen und das Gesicht überhaupt erst als Filmgesicht konstituieren.
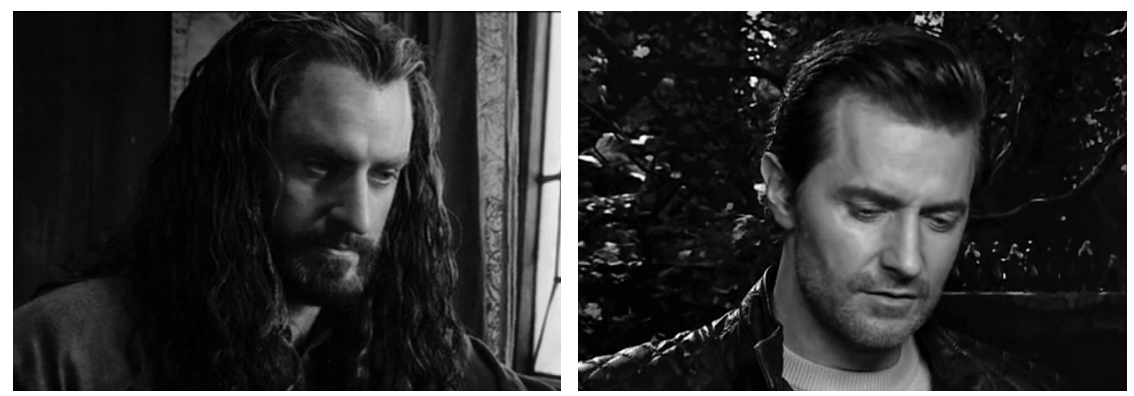

Abb. 1 und 2: Richard Armitage als Thorin Oakenshield in The Hobbit: The Desolation of Smaug (NZ/GB/USA 2014, R.: Peter Jackson) ${ }^{8}$ und in einem Interview während der Promotion-Tour zu The Desolation of Smaug ${ }^{9}$

Die Maske kann zudem die Grundspannung der Gesichtsmuskulatur als dem »mimischen Gelände ${ }^{10}$ des Antlitzes und seiner Beweglichkeit überdecken, Proportionen z.B. von Stirn und Nase verändern, ${ }^{11}$ die Schattenwirkungen von Falten manipulieren etc. Gleichwohl folgt bei den Hauptrollen

7 Ebd., S. 203.

8 Screenshot aus der DVD des Films.

9 Screenshot aus dem Interview mit Richard Armitage (2013), veröffentlicht auf : www. youtube.com/watch?v=20zfm7xRPBI (20. Februar 2016).

10 Claudia Schmölders: Das Vorurteil im Leibe. Eine Einführung in die Physiognomik, 3. Aufl., Berlin 2007, S. 135.

11 Vgl. die Maske von Nicole Kidman als Virginia Woolf in The Hours (Regie: Stephen Daldry, GB 2002). 
das Maskenbild (zumal im Horror- oder Fantasy-Genre) tendenziell dem Prinzip, das reale Gesicht des Schauspielers ’hinter dem Gesicht seiner Figur noch transparent bleiben zu lassen. Dies ist sowohl bei Gary Oldman in Francis Ford Coppolas Bram Stoker's Dracula (USA 1995) der Fall als auch bei Richard Armitage in The Hobbit, um nur zwei prominente Beispiele zu nennen. ${ }^{12}$

\section{Casting-Konventionen}

Nach welchen impliziten oder auch expliziten Normen verlaufen Auswahlprozesse zur Besetzung von Rollen beim Film und in welchem Maße sind diese Auswahlprozesse durch bewusst oder unbewusst angewandte, auf dem sunsicheren Wissen der Physiognomik beruhende Spekulationen der Filmakteure grundiert? - Als alltägliches Medienhandeln hat das Casting das Verhältnis zwischen tradierten Stereotypen über den Konnex von körperlicher Erscheinung und Charakter einerseits und andererseits Versuchen, sich bei der Rollenvergabe von diesen Stereotypen abzusetzen, auszubalancieren. Besetzungspraktiken bei Film und Fernsehen sind häufig vom Wissen um die bis dahin von den Schauspielern verkörperten Rollen und ihre damit eng verbundenen öffentlichen Images geprägt. Im entscheidenden Schritt zur Realisation eines bis zu diesem Zeitpunkt snur auf dem Papier existierenden Films greifen Vorstellungen bei den Verantwortlichen, die zwischen lektüregeleiteter Phantasie und gegebenenfalls schon im Vorfeld des Castings existierenden Besetzungsvorstellungen changieren. Im Starkino herrscht zudem die Praxis des Umschreibens und der Anpassung einer Figur, sollte das Skript Dialoge oder Handlungselemente enthalten, die nicht mit dem Image des besetzten Schauspielers kompatibel erscheinen. So schrieb etwa Hans Jacoby für Heinz Rühmann Passagen von Friedrich Dürrenmatts Drehbuch zu Es geschah am hellichten Tag (Regie: Ladislaus Vajda, CH/BRD/E 1958) um, indem er sie abschwächte, da sich im Original Handlungsweisen und Dialoge der Hauptfigur Kommissär Matthäi fanden, die Rühmann so nicht

12 Armitage sagte über seine faciale Verwandlung zu Thorin, für die er an jedem Drehtag ca. zweieinhalb Stunden in der Maske sitzen musste, dass sie ihm trotz der aufwendigen Stirn- und Nasenprothese die Möglichkeit bot, seine Augenbrauen zu bewegen und damit auch ein wandlungsfähiges, kontrollierbares Augenspiel zu performieren. Sein Bart war ebenfalls secht<. Die Vorentwürfe zu Thorins facialem Erscheinen waren ungleich verfremdender (und ließen die Figur auch deutlich älter erscheinen) als diejenige Maske, die schließlich für den Dreh verwendet wurde. 
zu spielen bereit war. ${ }^{13}$ Insbesondere die Art, wie Matthäi in Dürrenmatts Originalfassung des Skripts das kleine Mädchen als Lockvogel benutzt und er nur dem Ziel folgt, den Mörder zu fangen, widersprach Rühmanns Image, das er sich durch seine bis dahin gespielten Rollen (zumal in der Interaktion seiner Figuren mit Kindern) erworben hatte. ${ }^{14}$

Im Hinblick auf die operative Wirksamkeit physiognomischen Denkens im Filmskript ist Paul Schraders Drehbuch zu Taxi Driver (Regie: Martin Scorsese, USA 1976) ein gutes Beispiel. Sein Schreibverfahren zur ersten äußeren Charakterisierung der Hauptfigur Travis Bickle umspielt das Figurenäußere kalkuliert vage und ist zugleich detailreich:

Travis Bickle, age 26, lean, hard, the consummate loner. On the surface he appears good looking, even handsome; he has a quiet steady look and a disarming smile which flashes from nowhere, lighting up caused by a live of private fear, emptiness and loneliness. He seems to have wandered in from a land where it is always cold, a country where the inhabitants seldom speak. The head moves, the expression changes, but the eyes remain ever-fixed, unblinking, piercing empty space. [...] He has the smell of sex about him: Sick sex, repressed sex, lonely sex, but sex nonetheless. ${ }^{15}$

Nur selten haben sich Regisseure so explizit zur Besetzung einer Filmrolle geäußert wie Sergej M. Eisenstein, der für seinen Film Die Generallinie/ Das Alte und das Neue (SU 1927-1929) lange nach einer Darstellerin für die Protagonistin, eine junge Bäuerin, gesucht hatte und sie schließlich in der Person Marfa Lapkinas fand. Angesichts des dem Film zugrunde liegenden Originalstoffs konzentrierte sich die Besetzung der Hauptrolle primär auf die Referenzdimension zur zeitgenössischen Realität. Es galt eine Darstellerin zu finden, die aus der Sicht der Filmemacher glaubhaft eine verelendete Bäuerin verkörpern konnte - freilich eine, die sich im Verlauf des Films zu einer Figur mit Vorbildfunktion entwickelt. Dies bedeutete, dass sie mit ihrem Spiel die angezielten Publika davon überzeugen sollte, dass nicht nur die technische Modernisierung der Agrarproduktion das Gebot der Stunde ist, sondern auch die Kollektivierung der Landwirtschaft.

13 Vgl. die verschiedenen (nicht veröffentlichten) Drehbuchfassungen von Es geschah am hellichten Tag. Ich danke dem Aufnahmeleiter des Films, Philippe Dériaz, für die Einsichtnahme.

14 Vgl. zu Heinz Rühmann: Stephen Lowry/Helmut Korte: Der Filmstar, Stuttgart/Weimar 2000.

15 Paul Schrader [1976]: Taxi Driver. Drehbuch, London 1999, S. 1. Ob Schrader bei seiner Beschreibung von Travis Bickles den Darsteller Robert de Niro gleichsam schon rim Kopf hatte, muss hier unberücksichtigt bleiben. 

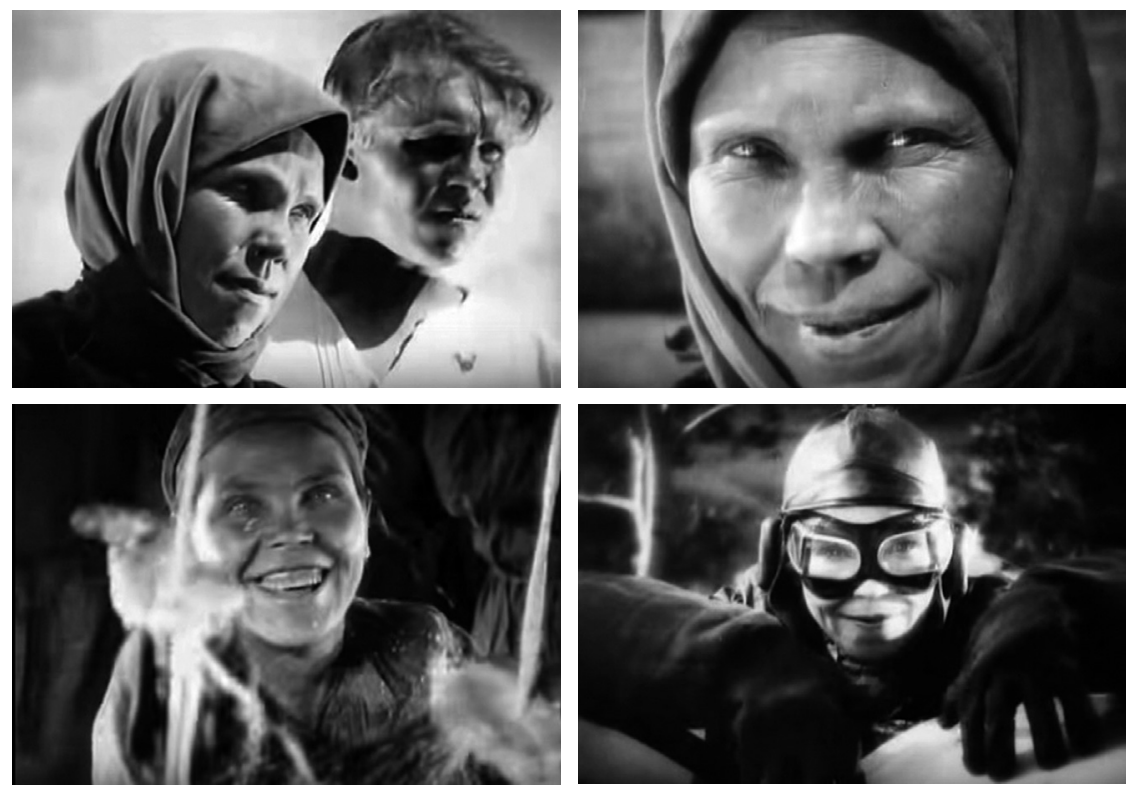

Abb. 3-6: Die Wandlungen Marfa Lapkinas im Verlauf des Films Die Generallinie/Das Alte und das Neue, 1929

Eisensteins in den 1920ern Jahren geübte Praxis, seine Filme hauptsächlich mit Laiendarstellern zu besetzen, war exemplarisches type casting. Er war auf der Suche nach `Durchschnittsmenschen dem Ziel, zwischen Leinwand und Zuschauerschaft eine möglichst große kommunikative Schließung zu erreichen, d.h., das adressierte Publikum sollte sich in der äußeren Gestalt der Arbeiter, Matrosen oder Bäuerinnen als neuen kollektiven Subjekten der geschichtlichen Entwicklung in Russland wiedererkennen. ${ }^{16}$ Dies veranlasste ihn dazu, keine professionellen Schauspieler für diese Figuren zu besetzen, sondern sich im sozialen Raum

16 Für den in Zürich gedrehten Film Frauennot - Frauenglück (CH 1929) stellte Eisensteins Kameramann Eduard Tissé, der bei diesem halbdokumentarischen Film die Regie übernahm, einen Cast aus Laiendarstellern und Schauspielern vom Zürcher Schauspielhaus zusammen. Vgl. zur Analyse des Films Ursula von Keitz: Im Schatten des Gesetzes. Schwangerschaftskonflikt und Reproduktion im deutschsprachigen Film 1918-1933, Marburg 2005, S. 280-286. Die Methode des kombinierten Casts auch bei der Besetzung der tragenden Rollen ist ein Markenzeichen russischer Filmemacher in den 1920er und frühen 1930er Jahren. 
außerhalb von Film und Theater umzusehen. ${ }^{17}$ Die Suche nach einer Hauptdarstellerin für Die Generallinie/Das Alte und das Neue gestaltete sich höchst aufwendig:

Die meisten Probleme brachte die Kandidatenauswahl für die Hauptrolle der Generallinie, weil mit ihr eine ganze Reihe hochkomplizierter Schauspielaufgaben verbunden war. [...] Nach Sichtung der ersten dreitausend Frauen (im Arbeitsamt, Übernachtungsheimen und auf Bauernversammlungen) hatten wir endlich unsere Vorstellung vom gewünschten Typus verloren und verfielen so in einer Art Kollektivpsychose auf irgendeine Frauenperson aus dem Moskauer Arbeitsamt, die freilich ihrem äußeren Erscheinungsbild nach ausgesprochen fotogen war. Doch gleich bei den ersten misslungenen Aufnahmen beschlichen uns schreckliche Zweifel, und ich ließ sie für alle Fälle mit dem Rücken zur Kamera filmen. [...] Und als dann schließlich sämtliche Möglichkeiten von 'Rückenaufnahmen ausgeschöpft waren, schickte uns >das Schicksak zwei Tage vor unserer Abfahrt nach Süden die Lapkina. [...] [Es wurde] unter den gerade pausierenden ZuchtgutsArbeiterinnen ein erstbester Rücken gewählt. Ja, und mit diesem Rücken kam nun Marfa Lapkina - unser >Dorf-Star< - zum Film. ${ }^{18}$

\section{Das lächelnde Gesicht der Marfa Lapkina, so Wolfgang Beilenhoff,}

erleuchtet nicht von elektrischen Scheinwerfern, sondern vom Sonnenlicht - [stellt] die Schönheit des Alltäglichen aus, die Welt vor dem Film, die Welt der Geschichte, die zu reflektieren das Kino sich immer wieder erneut anschickt. Es ist ein von der Geschichte geprägtes, auf die Geschichte antwortendes Lächeln [...]. ${ }^{19}$

17 Dass dieser Findungsprozess zu existentiellen Krisen führen kann, hat Michelangelo Antonioni in seinem Film Identificazione di una donna (I 1982) exemplarisch vorgeführt, wobei er das Verhältnis von Buch und Figur, Fiktion und Referenz gleichsam umdreht: Der Regisseur Niccolo kann seinem Drehbuchmitarbeiter Mario zwar das Thema seines nächsten Films nennen - eine Frau; aber es gelingt ihm nicht, in den Frauenporträts über seinem Schreibtisch die Konturen einer Geschichte zu entdecken. Vgl. hierzu Claudia Lenssen: Identificazione di una donna, in: Peter W. Jahnsen/Wolfram Schütte (Hg.): Michealangelo Antonioni, München/Wien 1987, S. 217-234.

18 Sergej M. Eisenstein: Das Alte und das Neue (Die Generallinie). Mit den Notaten eines Vertonungsplanes und einem Briefwechsel mit Wilhelm Reich im Anhang, hg., übers. und kommentiert von Hans-Joachim Schlegel, München 1984, S. $134 f$.

19 Wolfgang Beilenhoff: Lächeln, in: Gesichter des Films, hg. von Johanna Barck und Petra Löffler, Bielefeld 2005, S. 172. 


\section{Physiognomie und Pathognomie}

Die mit der Häufigkeit filmischer Großaufnahmen auftretende Präsenz des Gesichts bereits von der Stummfilmepoche an unterstreicht die elementare Bedeutung facialer Merkmale und mimischer Ausdrucksqualitäten. ${ }^{20}$ Carl Michels Die Gebärdensprache dargestellt für Schauspieler sowie Maler und Bildhauer von 1886 unterscheidet 94 verschiedene Ausdrucksqualitäten des Gesichts und benötigt zur Übersetzung seiner in Fotografien festgehaltenen Ausdrucksvarianzen mehrwertige Klassifizierungstermini aus Verb und Adjektiv wie »dummdreistes Lachen«, "schadenfrohes Lachen« o. Ä.

Indem Michel die verschiedenen emotiven Felder kartografiert und die ihnen zugrunde liegenden Muskelkontraktionen beschreibt, führt er ein Lernprogramm des nuancierten Zuschreibens ein, das sich für die Nachahmung wie für das interpretierende Verstehen der mimischen Differenzen gleichermaßen empfiehlt.
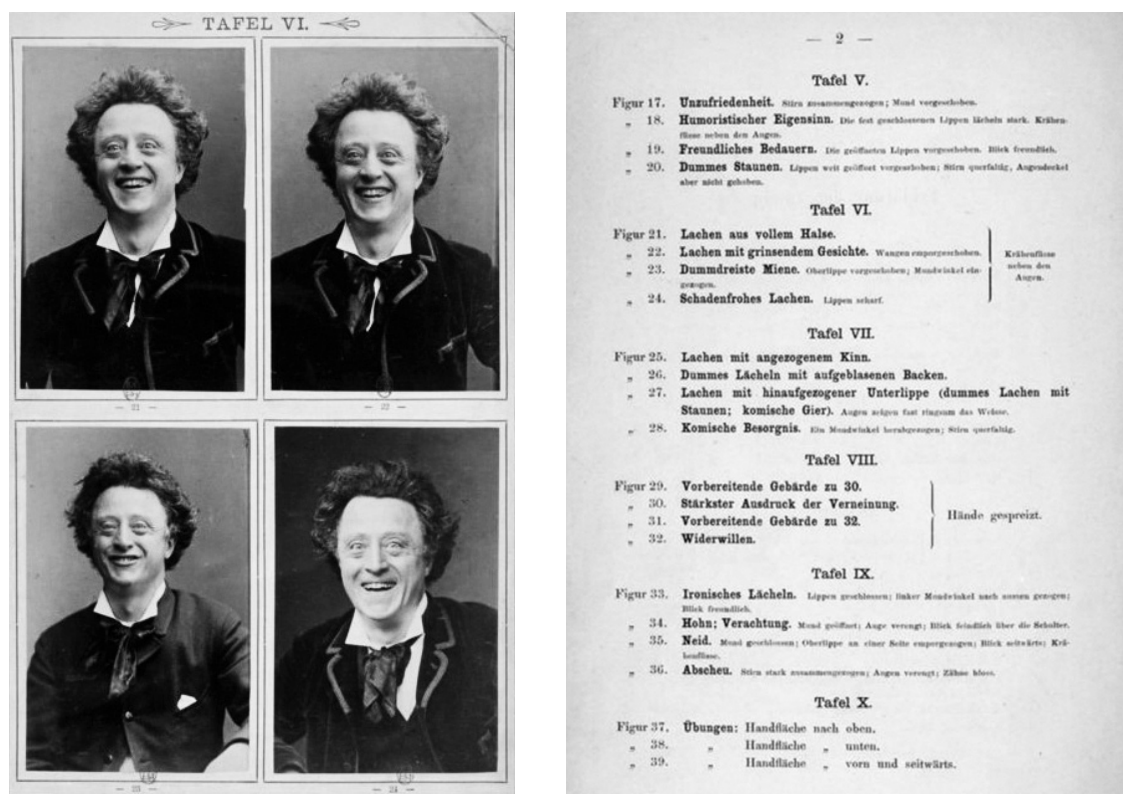

Abb. 7 und 8 aus: Carl Michel: Die Gebärdensprache dargestellt für Schauspieler sowie Maler und Bildhauer, 1886

20 Vgl. Jörg Schweinitz: Film und Stereotyp. Eine Herausforderung an das Kino und die Filmtheorie, Berlin 2006, S. 65-68. 
Während die physiognomische Vielfalt der Darsteller sehr groß ist, sind Schauspiellehren wie diejenige Michels darauf aus, die pathognomische Vielfalt im Sinne der Herausarbeitung einer möglichst ambivalenzfreien Performanz von Grundformen und Nuancen des Gesichtsausdrucks einzuschränken. Je eindeutiger sich mithin der emotionale Ausdruck auf dem Gesicht abbildet, desto leichter wird es gemäß diesem an den Wahrnehmungsbedingungen der Bühne sich orientierenden Leitfaden lesbar bzw. für das Publikum verstehbar. Dem voraus gehen indes physiognomische Basisannahmen darüber, dass die radäquate Verkörperung einer Figur auch von Grundmerkmalen des Gesichts, des Alters und der Statur der Darstellenden abhängt. Anhand jener Eigenschaften wurden sie in einen Katalog von Rollenfächern eingeordnet wie z.B. die jugendliche Naive‘, die 'Salondame oder die 'komische Alter. Die Rollenfächer lösten die strengen Figurentypisierungen in den Theaterstücken ab, die im Laufe des 18. Jahrhunderts sukzessive überwunden wurden. Sie bezogen sich nun auf ein Spektrum von Theaterrollen, auf das sich Schauspielerinnen und Schauspieler spezialisierten. Dabei hatte jedes Rollenfach festgelegte Ausdrucksmittel. Erst mit dem Naturalismus lösten sich dann auch die Fächer zugunsten einer individualisierten Gestaltung zunehmend auf. ${ }^{21}$

Den frühen, rein auf die mimischen und gestischen Ausdrucksmittel setzenden Film hingegen dominiert eine weitgehend schematisierte Besetzungspraxis; die Reduktion auf Typen und soziale Rollen wie sder Liebhaber, >der Gangster`, >der Lüstling«, ’der Arbeiter`, ’der Aristokrat‘ etc. wirkt einer Individualisierung zunächst in gewisser Weise entgegen. Dieser Besetzungsmodus hat viele Schauspieler in ein enges Korsett gezwängt, aus dem sie sich selten befreien konnten. ${ }^{22}$ Umso unerwarteter erschien z.B. Max Ophüls' gelungene - Besetzung von Luise Ullrich (als Mizzi) und Magda Schneider (als Christine) entgegen ihrer Fächer in seiner Schnitzler-Adaption Liebelei (D 1933). ${ }^{23}$

Filmcasting unterliegt historisch vor allem in organisatorischer Hinsicht einem Wandel, parallel zur immer stärkeren Ausdifferenzierung des Mediensystems. Die wichtigsten Zäsuren dürften hier (neben dem Übergang vom Stummfilm zum Tonfilm) die Auflösung des Hollywood-Studio-Systems mit

21 Vgl. Gerhard Ebert: Der Schauspieler. Geschichte eines Berufs, Berlin 1991.

22 Vgl. etwa zu Fritz Kortner: Martin Girod: Beredte Stummheit. Fritz Kortner als Filmschauspieler 1915-1929, in: Das Gedächtnis des Films. Fritz Kortner und das Kino, hg. von Armin Loacker, Wien 2014, S. 99-122.

23 So spielte Luise Ullrich bis dahin vor allem tragische Figuren, während Magda Schneider auf heitere, naive Figuren abonniert war. 
vertraglich mehr oder weniger lang gebundenen Schauspielern in den 1950er Jahren, die Lockerung der Zuordnung von Schauspielern zu Rollenfächern, die durch die Wahrnehmungsbedingungen des Fernsehens verstärkte Tendenz zu kleineren Einstellungsgrößen sowie seit den 1980er Jahren die Vermehrung des TV-Programmangebots insgesamt sein.

Casting-Büros treffen heute bei der Besetzung von Kino- und Fernsehfilmen, die im Auftrag von Produzenten oder Senderredaktionen erfolgt, eine nach Stereotypen-Kriterien verfahrende Auswahl in der Regel nur für die Nebenrollen. Schauspielerinnen und Schauspieler werden mit ihren besonderen Eigenschaften, Fähigkeiten und ihrer beruflichen Biografie in Karteien bzw. Datenbanken geführt, die beständig à jour gehalten werden. ${ }^{24}$ Für die Shows und Serien des Reality-TV werden in den letzten Jahren überdies Laiendarsteller als sogenannte social actors sbesetzt<.

Publikationen über Casting haben primär die Schauspielerinnen und Schauspieler selbst als Zielgruppe. Diesen geben sie, wie etwa Ginger Howard ${ }^{25}$ oder Ulrike Boldt, Ratschläge für die Vorbereitung von Vorsprechterminen und Verhaltensregeln im Umgang mit Besetzungsbüros und Castern. Welchen impliziten Annahmen über die `Passung` zwischen der körperlichen Erscheinung eines Darstellers und der ge- bzw. beschriebenen Figur im Drehbuch Caster als sSchwellenakteure folgen, ist bislang kaum erforscht. Boldt zitiert immerhin einige Kriterien, die Casting Directors bei der Besetzung von Hauptrollen berücksichtigen, so etwa ob die Schauspielerin oder der Schauspieler prominent genug ist, um Zuschauer ins Kino oder vor den Fernseher zu locken, ob sie oder er zur Rolle, zum Format, zum Sender und seinen Programm passt, ob sie als Sympathieträger(innen) geeignet sind und ausreichend Ausstrahlung besitzen, ob sie eine Figur über einen hinreichend langen Zeitraum glaubwürdig und spannend tragen können, ob sie das dazu benötigte schauspielerische Talent und die nötige körperliche Präsenz mitbringen usw. ${ }^{26}$

Die zweite Bedingung geht über den Aspekt der bloßen `Passung` zwischen dem Körper des Schauspielers und den im Drehbuch festgehaltenen Figureneigenschaften weit hinaus. Denn Format und Sender werden wie Marken geführt, für deren Identität nicht nur Programmarten, sondern (ähnlich wie

24 Vgl. die Interviews mit Casting-Agentinnen und -Agenten in: Ulrike Boldt: Casting für Film, Fernsehen und Bühne. Der Wegweiser zum Erfolg, Berlin 2008, S. $47 \mathrm{ff}$.

25 Ginger Howard: Casting Director's Secrets. Inside Tips for Successful Auditions, New York 2000.

26 Vgl. Ulrike Boldt: Casting für Film, Fernsehen und Bühne, S. 44. 
im klassischen Studiosystem) auch `Gesichter einzustehen haben. Damit ist impliziert, dass Aspekte wie Individualität oder spezifische, mit der Verkörperung verschiedener Figuren jeweils wechselnde Stile zugunsten der Referenz auf eine Format-, Programm- oder auch Senderidentität, über deren relative Konstanz die Redaktionen wachen, letztlich zurückzutreten haben.

Joseph Turow führte 1978 Interviews mit Autoren, Produzenten, Regisseuren, Schauspielagenten und Castern in Los Angeles, um sie für seine Studie Casting for TV Parts: The Anatomy of Social Typing auszuwerten. ${ }^{27}$ Der Besetzungsmodus bei den Nebenfiguren, die nur wenige Szenen und ein paar Seiten Dialog haben, ist für Turow deshalb interessant, weil deren äußere Erscheinung häufig (worauf auch Tucker hinweist) durch keine nähere Beschreibung im Drehbuch konkretisiert ist. Mithin sind die Vorstellungsspielräume für die Besetzung prinzipiell groß. Der Casting Director stellt eine mögliche Liste von Darstellern zusammen und bespricht sie mit Produzent und Episoden-Regisseur. Die ausgewählten Darstellerinnen und Darsteller werden zu Lesungen eingeladen. Alle haben im Blick, unterhaltsame Geschichten erzählen zu wollen, die in einer visuell ansprechenden Weise ein Millionenpublikum erreichen sollen. Zwei Kriterien leiten ihre Auswahl: `Glaubwürdigkeit`(credibility) und `optische Ausgewogenheit< (visual balance). Erstere betrifft die Ansicht des Casters über die Mutmaßungen der meisten Menschen darüber, wie jemand aussieht, der einem bestimmten Beruf nachgeht oder eine soziale Rolle innehat; Letztere seine Ansicht darüber, wie ein Schauspieler zum anderen in ästhetischer Hinsicht passt. Die Caster geben indes zu, nicht zu wissen, was die meisten Leute denken, und setzen daher ihre eigenen Vorstellungen oder die ihrer Kollegen kurzerhand als Norm. Einige behaupten, die Kriterien für die Glaubwürdigkeit seien seit den ersten Tagen des Films bei Besetzungsentscheidungen in den USA konstant geblieben. ${ }^{28}$ Was das Publikum der Szene sofort und ohne Umschweife akzeptiert, gibt die Orientierung vor. Dies gilt ebenso auch für ethnische wie geschlechterspezifische Aspekte: »Why make a point out of something that's not a point? Carpenters are male. Telephone operators are female. Now, there are a lot of male telephone operators now. But not enough so that when you say stelephone operator<, the immediate thought isn't female. ${ }^{29}$ Kriterien

27 Joseph Turow: Casting for TV Parts: The Anatomy of Social Typing, in:Journal of Communication 28/4 (Autumn 1978), S. 18-24, hier S. 19. Alle von Turow befragten Personen waren weiße Männer. »The dominant perspectives, requirements, and relationships direct the activities of everyone involved«, so Turows kritisches Resumée (ebd.).

29

Vgl. ebd., S. 20.

Ebd. 
sind der Kontrast, eine nicht zu große Ähnlichkeit im Erscheinungsbild und eine gegengeschlechtliche attraktive Person, die der Hauptdarsteller oder die Hauptdarstellerin anspielen kann. Gegen einen solchen Typ bzw. gegen ein Klischee zu besetzen, kann der Szene mehr Tiefe verleihen, doch meist unterbleibt dies wegen des eng gesteckten Zeitrahmens für die Besetzung. Serienprogramme werden ihrerseits hinsichtlich ihrer Erzählstile und Looks klassifiziert, unterteilt in beautiful people shows, real people shows und street people shows. ${ }^{30}$ Die sich selbst immer wieder bestätigenden Besetzungskonventionen im US-Fernsehen beschreibt Turow als Resultat einer von Produzenten, Regisseuren, Schauspielagenten und Castern geteilten dominanten Sicht auf Gesellschaft. ${ }^{31}$ Diese Branchenvertreter haben sich im Raum Los Angeles niedergelassen, sie rezipieren auch ihrerseits das Fernsehen und kennen ihre gegenseitigen Vorlieben. ${ }^{32}$ Gemäß den von Turow herausgearbeiteten Einstellungen der entscheidenden Akteursgruppen werden bei Rollenbesetzungen demnach kaum Entscheidungen getroffen, die der Glaubwürdigkeitsdirektive widersprechen. Zur Glaubwürdigkeit wiederum gehört nicht nur die 'Passung zwischen der Körperlichkeit der Schauspielerin oder des Schauspielers, ihrem Gesicht und ihrer Statur zur fiktiven Figur bzw. ihrer sozialen Rolle und deren außerfilmische Referenzialisierbarkeit, sondern letztlich auch die Bestätigung der beim Publikum vorhandenen Zuschreibungen bestimmter Eigenschaften zu gewissen Körpertypen.

Der eingangs zitierte Patrick Tucker problematisiert das type casting, das auch bei größeren Rollen greift und mit einer Nachhaltigkeit die filmischen Besetzungskonventionen prägt, die kaum durchbrechbar erscheint:

30 Unschwer lässt sich diese Klassifikation bis heute bei US-Serien erkennen, vergleicht man etwa die Körperlichkeit der Darsteller in Mad Men mit der von The Wire.

$31 \mathrm{Ob}$ sich einerseits aufgrund der heute vermehrt von Frauen ausgeübten Casting-Praxis, andererseits aufgrund des gesellschaftlichen Wandels seit den 1970er Jahren Verschiebungen ergeben haben, wäre zu untersuchen.

32 Wenn soziale Gruppen außerhalb der Industrie eine Öffnung des Besetzungsspektrums sozialer Charaktere mit Blick auf Gender oder Ethnizität fordern, so kommen zwar bisweilen minoritäre Entscheidungen zustande. Aber im Falle etwa der Besetzung eines jungen, afroamerikanischen Darstellers als Pfleger (statt der ursprünglich gewünschten Krankenschwester) in einer Hospital-Serie wurde peinlich darauf geachtet, dass die Zahl der Minderheiten- zur Zahl der Mehrheitsdarsteller, "6 non-whites and 12 whites " nicht zu hoch war. Vgl. Joseph Turow: Casting for TV Parts, S. 23. - Aktuell wird die Besetzungspolitik in Deutschland, insbesondere was Gender-Aspekte betrifft, kritisiert und eine `Neutralrollenparität für Frauen und Männer gefordert. Vgl. http://neropa.stieve.com (20. Februar 2016). Besetzungspraktiken für Film und Fernsehen hinken, so die Kritik, dem Gesellschaftswandel faktisch hinterher. 
We all admire and want to be those actors who do portray many different character types, even with different accents (Robert de Niro, Ewan McGregor, Gwyneth Paltrow, Meryl Streep), but we end up - if we are very lucky - playing the same roles as do Julia Roberts and Bruce Willis. It is simple economics. A film is so very expensive, the last thing the money people want is to take a risk with a performance, so they buy the performance that they already know the public likes. ${ }^{33}$

Infolgedessen empfiehlt er Darstellerinnen und Darstellern, aus ihrem Typus das Beste zu machen - und dies beginne damit, sich mit diesem Typus möglichst vertraut zu machen:

Look in the mirror, and be firm and realistic. What message comes across from your faces in repose? Now that is what the message of your performance will be, and it will owe more to the packet of genes given to you by your parents than to the artistic/creative acting process you went through to prepare for the role. ${ }^{34}$

Auch bei der Besetzung des Hauptcasts wird indes ein implizites >Wissen aktualisiert, das die Physiognomik als popularisierte, spekulative Episteme zur Grundlage hat. Dabei lässt sich ein Zuschreibungsfundament unterstellen, das von Lavaters Projekt einer Charakterdeutung aus unveränderlichen, anatomischen Gegebenheiten des Gesichts, aus der Beschaffenheit von Stirn, Nase, Augen, Augenbrauen, Mund, Zähnen etc. über Carl Huters PsychoPhysiognomik ${ }^{35}$ und Ernst Kretschmers Konstitutionstypenlehre ${ }^{36}$ bis hin zu William Sheldons Somatotyping ${ }^{37}$ reicht. Sognannte sendomorpher Charaktere sah Sheldon als entspannte Menschen, die dem Kulinarischen zugeneigt seien und Entscheidungen intuitiv fällten. Die muskulösen `Mesomorphen hielt er für körperlich fit, voller Energie, mutig und selbstsicher. Die langen,

33 Patrick Tucker: Secrets of Screen Acting, S. 86. Hervorhebungen im Original fett gedruckt.

34 Ebd., S. 84.

35 Vgl. Carl Huter: Grundlegende Entdeckungen für die wissenschaftliche Psychophysiognomik, Leipzig 1910.

36 Vgl. Ernst Kretschmer: Körperbau und Charakter. Untersuchungen zum Konstitutionsproblem und zur Lehre von den Temperamenten, Berlin 1921. Hier figurieren die Differenzen (vor allem männlicher Körperbautypen) als sathletisch`, spyknisch und ’leptosom‘.

37 William Herbert Sheldon/Stanley Smith Stevens: The Varieties of Temperament: A Psychology of Constitutional Differences, New York 1942. Sheldon wollte eigentlich Kretschmer widerlegen, kam aber in einer empirischen Untersuchung zu ähnlichen Ergebnissen, als er 1940 in einer Testreihe die körperlichen Merkmale von 4000 Studenten untersuchte. Er führt die Entwicklung der körperlichen Konstitution auf die drei Keimblattgewebe des Embryos zurück, die jeweils verschieden stark ausgeprägt sein können. Ein wesentlicher Kritikpunkt an der Arbeit Sheldons richtet sich gegen seine Methode der Merkmalserfassung; denn Sheldon führte keine Messungen im eigentlichen Sinne durch, sondern entwickelte eine standardisierte Auswertung von Körperbaufotos. 
dünnen `Ektomorphen` hingegen seien eher kopflastige, künstlerische und introvertierte Menschen, die mehr über das Leben nachdächten, als dass sie es ausschöpften oder gestalteten.

Sheldons Konzeption der Somatotypen basierte auf implizitem Alltagswissen, welches, einmal als Theorie formuliert, zumindest in den USA wiederum auf die allgemein verbreiteten Annahmen über Körpertypen zurückwirkte. Die Übertragung einer weitgehend unreflektierten typologischen Betrachtung der menschlichen Statur auf die jeweiligen sKörpertypen` von Filmfiguren (und den damit verbundenen Zuschreibungen von Charaktereigenschaften) haben Cynthia Hoffner und Joanne Cantor 1991 in ihrer Rezeptionsstudie Perceiving and Responding to Mass Media Characters nachgewiesen. ${ }^{38}$ Die Autorinnen beschreiben,

welche Charaktereigenschaften Betrachter Personen allein aufgrund ihres Körperbaus zuschreiben: der "endomorphe« Typ wird als "lazier«, "talkative«, "warmhearted « und "generous" angesehen, der "mesomorphe« gilt als »strong «, "self-reliant « und sogar als »leader«, und der »ektomorphe« als "ambitious«, »suspicious«, "tense«, "nervous« und »stubborn«. 39

Gemeinsam ist Physiognomik und Somatotyping der Wunsch nach einem Hintergehen sozialer Akzidenzien wie Sprachverhalten, Status und Kleidung. Jegliche erlernte Fähigkeit oder erworbene Kenntnis wird letztlich als vortäuschbar ausgelegt. Der Wunsch nach einer Verständigung ohne Sprache, nach Beurteilungskriterien für Menschen, zu denen man gar nicht erst näher in Kommunikation treten muss, um sie schon erkannt und durchschaut zu haben, führt zum Motiv der Vorhersagbarkeit ihres Verhaltens in bestimmten Situationen. Dies wird gesellschaftlich da relevant, wo es um Schwellensituationen wie die Verteilung von Chancen, um Einschluss- und Ausschlusspraktiken z.B. bei Stellenausschreibungen geht, bei denen rauf dem Papier` zwar Fähigkeiten, Wissen und Kompetenzen der gesuchten Person abgefragt werden, in der Letztentscheidung aber vielfach das soptische Erscheinungsbild einer Kandidatin oder eines Kandidaten den Ausschlag gibt.

38 Cynthia Hoffner/Joanne Cantor: Perceiving and Responding to Mass Media Characters, in: Responding to the Screen. Reception and Reaction Processes, hg. von Bryant Jennings und Dolf Zillmann, Hillsdale (NJ) 1991, S. 63-102.

39 Annemone Ligensa: Stars und ihr Publikum am Beispiel von Clint Eastwood, Hamburg 2011, S. 154, Anm. 627. 
Die Arbeit des Castings als Ein- und Ausschlussverfahren, welches das Reich der Fiktionen mit konkret wahrnehmbaren sozialen Ensembles bevölkert, macht sich in der realen Welt existierende Zuschreibungspraxen zwischen physischer Konstitution und Charakter für fiktionale Figuren zunutze. Die alltagsreale physische Erscheinung einer Schauspielerin oder eines Schauspielers steckt dabei einen Erwartungshorizont (ein Versprechen) für die Glaubwürdigkeit der >Passung« zwischen der äußeren Erscheinung einer Figur und ihrem Charakter in der fiktionalen Welt ab. Und diese stereotypen physiognomischen Zuschreibungen können eine so große fiktionsleitende Dominanz bekommen, dass vom Drehbuch durchaus vorgesehene individuelle Physiognomien ver- und am Ende überformt werden.

\section{Die Besetzungsreihe von Rochester in den Jane-Eyre-Adaptionen von 1996, 2006 und 2011}

Anders als bei Originalstoffen müssen sich die Drehbücher von Adaptionen stets aktiv zum literarischen Prätext verhalten, indem sie sich in relativer Nähe oder Distanz zu diesem positionieren - und dies im Hinblick auf alle audiovisuell umsetzbaren narrativen Parameter. Dies gilt auch für Charlotte Brontës Roman Jane Eyre (1842). In jedem Skript, das als künstlerischer Folgetext eine Neu-Interpretation dieses Klassikers leistet, ${ }^{40}$ wird dabei eine je eigene Sicht auf dessen Normen, Werte und diskursive Darstellungspraktik entwickelt, wobei die Grundstruktur der Geschichte von Adaption zu Adaption erhalten bleibt. ${ }^{41}$ Zwischen einer stexttreuen Übernahme von

40 Kein englischer Roman des 19. Jahrhunderts dürfte wohl öfter filmisch adaptiert worden sein als Fane Eyre. Belegt sind ab 1919 (vgl. Die Waise von Lowood, D 1919, Regie: Carl Müller-Hegens, Drehbuch: Marie-Luise Droop) mindestens zwölf Verfilmungen für Kino und Fernsehen.

41 Hierzu gehören Janes von Ausgrenzung geprägte Lage als Waise im Haus ihrer verwitweten Tante Reed in Gateshead, ihre strenge Erziehung im Lowood Internat, ihre Freundschaft zu Helen Burns und deren früher Tod, der Wechsel nach Thornfield Hall zur Unterweisung Adèles, die schockhafte erste Begegnung mit Rochester, dessen Rettung vor dem Feuer im Bett durch Jane (nach Berthas Attacke), die verschworene nächtliche Pflege Masons (nach Berthas Attacke), Rochesters uneindeutige Beziehung zu Blanche Ingram, Janes temporäre Rückkehr nach Gateshead und die Nachricht von einem lebenden Onkel in Madeira (den sie später beerben wird), Rochesters Heiratsantrag, die durch Mason vereitelte Trauung, Janes Flucht, ihre Rettung und der Beginn einer neuen Existenz durch und bei St. John Rivers, dessen Missionspläne und Heiratsantrag, Berthas Tod und Rochesters Erblindung/Schädigung, Janes Rückkehr ins zerstörte Thornfield und schließlich nach Ferndean zur Vereinigung mit Rochester. 
Brontës Beschreibung der Figurenmerkmale - in unserem Fall Rochesters im Skript und einer Berücksichtigung dieser Merkmale auch im Castingprozess können weitere Differenzen entstehen, wenn etwa der für eine Rolle auch aufgrund seiner äußeren Erscheinung präferierte Darsteller nicht überzeugt und deshalb das Spektrum des Äußerlichen erweitert werden muss. Im Roman wird Rochester als Figur von kräftiger Statur, zornig-finsterem Ausdruck und schwarzem, widerborstigem Haar eingeführt. Die Ich-Erzählerin Jane arbeitet einen physiognomischen Merkmalskatalog zu dessen Beschreibung ab, deutet Rochesters Erscheinung aber spezifisch um im Hinblick auf ihre Art der Interaktion mit ihm. Über ihre erste Begegnung, bei der sein Pferd scheut und ihn abwirft, berichtet sie:

His figure was enveloped in a riding cloak, fur collared, and steel glasped; its details were not apparent but I traced the general points of middle height, and considerable breadth of chest. He had a dark face, with stern features and a heavy brow; his eyes and gathered eyebrows looked ireful and thwarted just now; he was past youth, but had not reached middle age; perhaps he might be thirty-five. Had he been a handsome, heroic-looking young gentleman, I should not have dared to stand thus questioning him against his will, and offering my services unasked. ${ }^{42}$

Gerade das Fehlen von Jugend und Schönheit veranlasst die Titelheldin, dem Gestürzten ihre Hilfe anzubieten. Männliche Schönheit brächte sie demnach eher in die Defensive. Es geht nicht darum, das äußere Erscheinungsbild zu neutralisieren, d.h. es für irrelevant zu erklären, sondern es umzuwerten und den (potentiell) abschreckenden Effekt, den Rochesters zorniges Gesicht hat, zu überwinden. Bei der zweiten Begegnung abends auf Thornfield Hall erleichtern seine Gesichtszüge und seine Statur das Wiedererkennen, wobei Kopfform und Körperbau nun näher beschrieben werden:

I knew my traveller with his broad and jetty eyebrows, his square forehead, made squarer by the horizontal sweep of his black hair. I recognized his decisive nose, more remarkable for character than beauty, his full nostrils, denoting, I thought, choler; his grim mouth, chin, and jaw - yes all three were very grim, and no mistake. His shape, now divested of cloak, I perceived harmonised in squareness with his physiognomy: I suppose it was a good figure in the athletic sense of the term - broad-chested and thin-flanked, though neither tall nor graceful. ${ }^{43}$

42 Charlotte Brontë: Jane Eyre. An Autobiography (1847), hg. von Richard N. Dunn, New York/London 2001, S. 96.

43

Ebd., S. 102. 
Mit der Negation von gleichsam rgelernten« Merkmalen männlicher Schönheit (»tall«, "graceful«) tritt an deren Stelle die sstimmige Aufeinanderbezogenheit von Gesicht und Statur als "good figure«. Der Katalog physiognomischer Elemente positioniert Rochester im Feld athletischer Männlichkeit. Und wieder unterläuft Jane mögliche, aus dessen Erscheinung und Grobheit resultierende Ängste. Er stößt weder auf Gegenwehr noch auf Eingeschüchtertsein, seine Grobheit prallt vielmehr an ihrer Verhaltenssicherheit ab. Die Analyse der Besetzungsreihe Rochesters in den Film- bzw. Fernsehadaptionen von 1996 mit William Hurt, ${ }^{44}$ von 2006 mit Toby Stephens ${ }^{45}$ und von 2011 mit Michael Fassbender ${ }^{46}$ möchte ich exemplarisch an der Szene durchführen, in der Rochester die junge Gouvernante danach fragt, ob sie ihn gutaussehend findet. Der Roman kontextualisiert diesen Dialog wie folgt:

Mr. Rochester, as he sat in his damask-covered chair, looked different to what I had seen him look before; not quite so stern - much less gloomy. There was a smile on his lips, and his eyes sparkled, whether with wine or not I am not sure; but I think it very probable. He was, in short, in his after-dinner mood; more expanded and genial, and also more self-indulgent than the frigid and rigid temper of the morning; still he looked preciously, cushioning his massive head against the swelling back of his chair, and receiving the light of the fire on his granit-hewn features, and in his great, dark eyes; for he had great dark eyes, and very fine eyes too - not without a certain change in their depths sometimes, which it was not softness, reminded you, at least, of that feeling.

He had been looking two minutes at the fire, and I had been looking the same length of time at him, when turning suddenly, he caught my gaze fastened on his physiognomy.

"You examine me, Miss Eyre«, said he, »do you think me handsome?»

$[\ldots] »$ No, sir.»

$[\ldots] »$ What do you mean by it?

"Sir, I was too plain. I beg your pardon. I ought to have replied that it was not easy to give an impromptu answer to a question about appearances; that tastes differ, that beauty is of little consequence, or something of that sort. « ${ }^{47}$

44 Jane Eyre, GB/I/F 1996, Regie: Franco Zeffirelli, mit Charlotte Gainsbourgh als Jane, Länge: 106 min., DVD, dt. Fs.

45 Jane Eyre, BBC 2006, Regie: Susanna White, mit Ruth Wilson als Jane, 4 Teile, Länge: 230 min., DVD, engl. Fs.

46 Jane Eyre, USA 2011, Regie: Cary Fukunaga, mit Mia Wasikowska als Jane, Länge: 115 min., DVD, engl. Fs. Die drei Adaptionen weisen eine Ähnlichkeit untereinander insofern auf, als sie alle auf Haddon Hall gedreht wurden. Zwar unterscheiden sich die Interieurs der einzelnen Räume, doch sind Gartenanlage und Gesamtarchitektur des Schlosses von Film zu Film wieder erkennbar.

Charlotte Brontë: Jane Eyre, S. 111f. 
Franco Zeffirellis Adaption von 1996 entfernt sich am weitesten von Brontës Textvorlage. Dies tritt nicht nur in der umfassenden Reduktion der durch die Modellierung des Lichts entstehenden, unheimlichen Wirkung der Innenräume des Schlosses zutage - jeder Raum ist gleichmäßig ausgeleuchtet, schattenfrei und tiefenscharf. Die Distanz zur Vorlage artikuliert sich vor allem auch in der Besetzung Rochesters durch den hellblonden, hoch aufgeschossenen Schauspieler William Hurt. Hurt erscheint in kaum einer Szene wirklich präsent, spielt den in die Jahre gekommenen Landadligen als verstörenden und verstörten Sonderling, der durch sein Anwesen wie ein Fremder wandelt. Zeffirelli situiert den zitierten Dialog nicht abends am Kamin, sondern am helllichten Tag im Garten. Adèle hat Jane gebeten, ihr eine Zeichnung ihres Ziehvaters, den sie sehr liebt, anzufertigen. Rochester wiederum hat registriert, dass Jane ihn aus der Ferne intensiv beobachtet, steigt die Terrassenstufen hinauf und sagt: "Sie haben mich beobachtet, Miss Eyre. Finden Sie mich gutaussehend? «48

Rochester steht in einer Halbtotalen, vorher jedoch wurde der Beginn des Zeichnens in einem Schuss-Gegenschuss zwischen ihm in der Nahen und Jane in der Halbtotalen dargestellt. Der Porträtierte schaut ernst und ruhig.
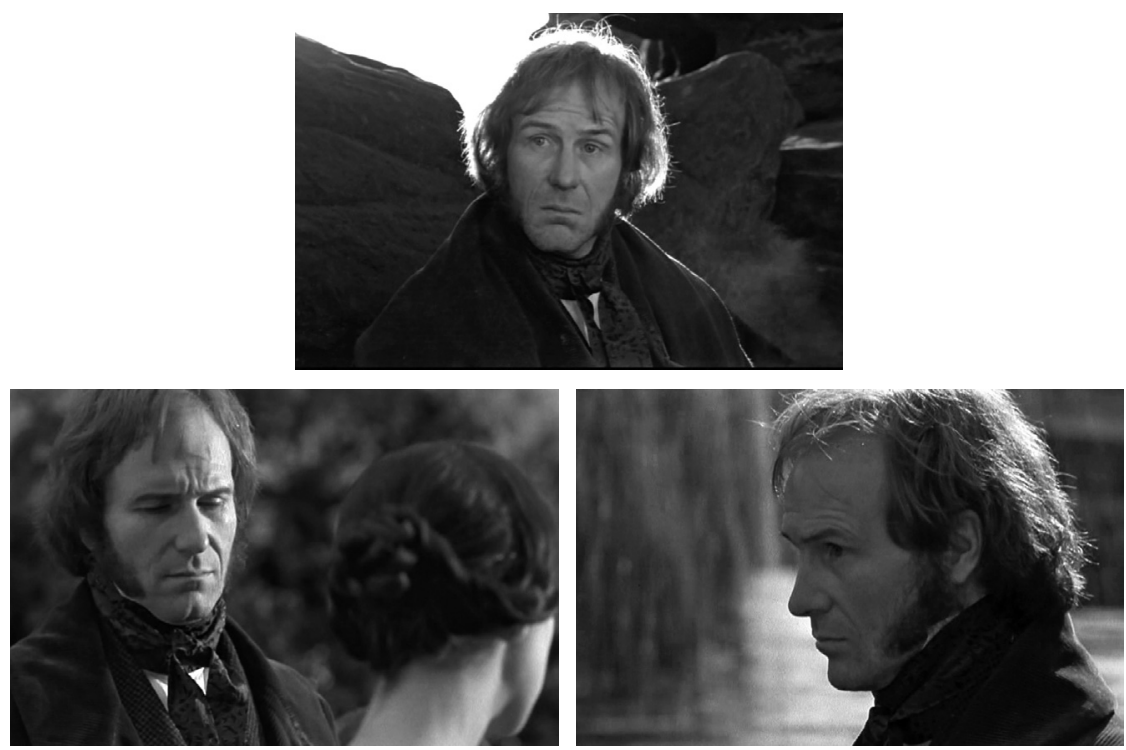

Abb. 9-11 William Hurt als Rochester, 1996

Zit. nach der deutschen Synchronfassung. 
Während des Schlagabtauschs bleibt der Abstand beider gewahrt, Umschnitte von Halbtotale zu Halbtotale unterstreichen die räumliche Distanz. Hörbar verärgert über ihre Antwort (»Nein«) will er deren Absicht erfahren. William Hurts Mienenspiel changiert zwischen Überraschung und Skepsis, da er nicht mit einer derartigen Antwort Janes gerechnet hat. Die Augenpartie bleibt unbeweglich, umso mehr kräuselt sich die Stirn, die Mundpartie mit den fest geschlossenen Lippen ist angespannt. Jane hat in ihrer Zeichnung Schatten über sein Gesicht gelegt, was Rochester sein eigenes Inneres‘ zu erkennen ermöglicht (»Sie haben mich gut getroffen...«). Er zerknüllt die Zeichnung, Adèle schreitet sofort dagegen ein.

Das Blickregime bei Zeffirelli verhindert, dass Jane und Rochester sich näher kommen, die Szene lenkt den Diskurs über die Lesbarkeit des Gesichts und seine Bewertung als "schön« um auf die Zeichnung (was zugleich auch die Differenz zwischen gefilmten und gezeichnetem Bild unterstreicht). Janes Blick modifiziert sein Profil, sie zeichnet, subjektivierend, Rochester weicher als das Gesicht Hurts, das die Kamera zuvor eingefangen hat. Die fortgesetzte Unbeweglichkeit der Augenpartie Hurts gibt der Figur durch die gesamte Inszenierung hindurch etwas Erstarrtes (Depressives), das, obwohl die Geschichte dies insinuiert, auch Janes Liebe nicht aufzulösen vermag. Im Vergleich zu Zeffirellis Adaption verfährt die vierteilige Verfilmung der BBC aus dem Jahre 2006 eindeutig texttreuer. Toby Stephens ist von kräftiger Statur und verfügt über eine hohe Körperspannung, er nimmt sich Raum und beherrscht ihn, schreitet energisch aus und geht breitbeinig durch Zimmer und Flure. Die halblangen schwarzen Haare sind leicht gewellt.

Dem Schlagabtausch darüber, ob er in Janes Augen »handsome" sei, der abends in seinem Arbeitszimmer stattfindet, geht ein Dialog voraus, in dem Rochester ihr eröffnet, sich in ihr als jungen Mann selbst wiederzuerkennen. "When I was a young man, I was your equal. (off) I had a clear conscience. I'm polluted by sin. (on) I took the wrong path. It wasn't my fault that I took it. But I must bare the blame for continuing on it." Diese Sätze sind aufgelöst in vier Nah- bzw. Großaufnahmen, jeweils unterbrochen durch Gegenschüsse auf Jane in Halbnah und Groß. Es beginnt in einer Nahen, wobei Stephens, ehe er zu sprechen beginnt, den Kopf etwas schieflegt und mild, leicht verträumt lächelt, dabei seine linke Mundhälfte etwas höher als die rechte zieht, wobei die Augenpartie mit erfasst wird. Die Augen leuchten, was dem Gesicht, gestützt auch durch die Brauntöne im Bild, Zärtlichkeit und Wärme verleiht. Dieser mimische Ausdruck, der nur für einen Moment anhält, trägt dazu bei, dass Stephens' Mund mit der herzförmigen Oberlippe, der ihm in einer semotionsneutralen Gesichtsmimik im Sinne Tuckers einen 

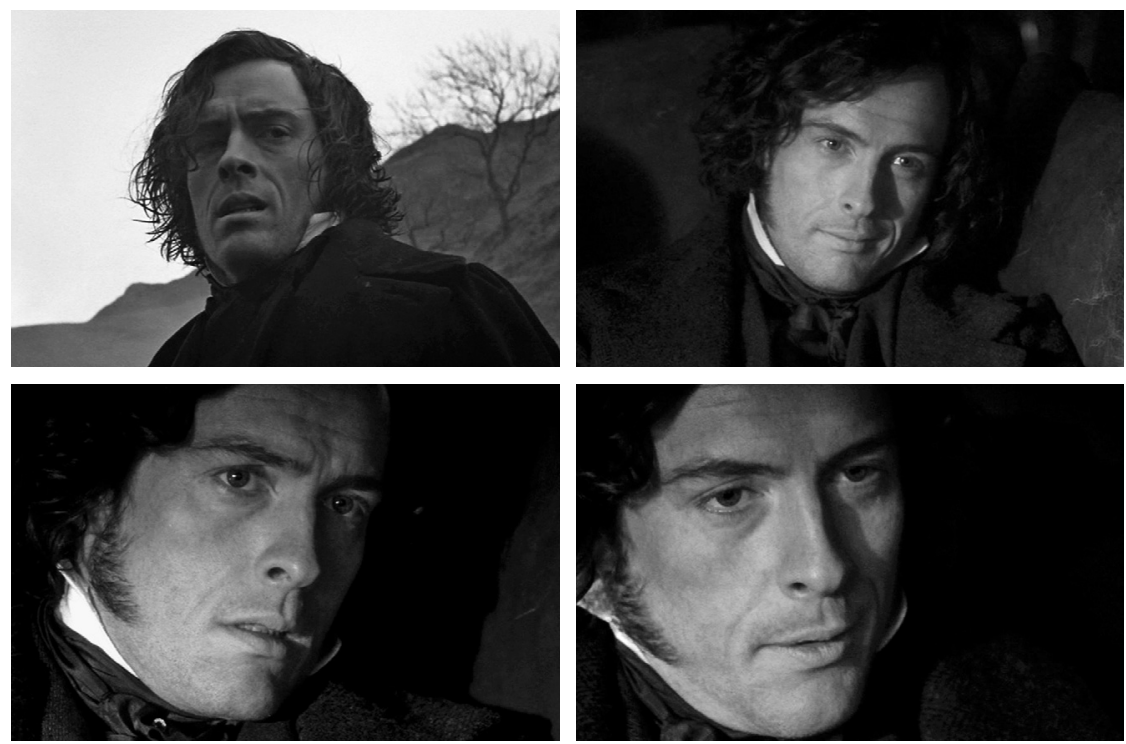

Abb. 12-15 Toby Stephens als Rochester, 2006

melancholischen Zug verleiht, das ganze Gesicht in seiner zarten Freude überraschend weich macht. Zugleich spielt er mit dem einleitenden Satz ein introvertiertes Schauen; er blickt zwar in Janes Richtung, schaut sie aber nicht eigentlich an. Es folgt ein Hin- und Herschneiden zwischen den beiden in Close-ups. Bei Rochesters Geständnis »I took the wrong path..." rückt die Kamera seinem Gesicht, dessen linke Hälfte nun im Halbdunkel fast verschwindet, in leichter Untersicht noch näher. Die Blickrichtung bleibt gleich, aber der Ausdruck hat gewechselt, vermittelt das Echo von Schuldgefühl und Erschrecken über sich selbst. Sofort wechselt die Stimmung zu einem bitteren Lächeln. Diesem folgt ein Umschwung in einen sein junges Gegenüber spielerisch-testenden und zugleich verführenden Modus, den Stephens mimisch ausgestaltet, bevor er fragt: "Do you think me handsome?" Als Reaktion auf Janes Antwort "No, Sir « lacht er kurz auf und steigert nun das verführerisch-testende Spiel, wobei das Gesicht noch näher kommt und er nachhakt: "Look. If I would tell you that I am worth $20.000 £$, sureley the light from the fire would soften my features. Do I not seem to you now the very model of a fashionable man?"

Stephens' Spiel mit der Kamera und das Blickregime des Films spannen in Susanna Whites Regie ein kommunikatives Dreieck auf, in dem die Zuschauer, anders als bei Zeffirelli, ihrerseits viel stärker in die »handsome«-Frage ein- 
bezogen werden. Sie beurteilen die schauspielerische Persona (mitsamt ihrer die Reaktionen austestenden Koketterie) durch die Figur 'hindurch', und so führt Stephens' bewegliche Mimik, mit der er die jähen Stimmungswechsel Rochesters gestaltet, die Zuschauerwahrnehmung durch die Szene. Jane ist sein lebendiger Spiegel, und seine Frage nach der 'Schönheit zieht sich bis zum Dialog in Ferndean hin, wo sie dem Erblindeten auch sein Auge wird (»It's a ghostly sight, isn't it, Jane?«) - und dies auch im Schlussbild bleibt, denn das Drehbuch gesteht Rochester hier keine Augenheilung zu. Die Diskussion über sein Aussehen legt hier nahe, dass Rochesters Selbstwahrnehmung auf einer tiefen Kränkung beruht. Sie erklärt sich durch seinen Status als nicht erbender Sohn und Objekt der Heiratsmachenschaften seines Vaters im Komplott mit der Familie Mason sowie durch seine mangelnde erotische Attraktivität (»unpleasant face«), die durch Céline Varens' Betrug nur umso mehr bekräftigt wird: $20.000 £$ make him very presentable.«49 Auch in Cary Fukunagas Version von 2011 mit Michael Fassbender sitzt Rochester in der »Do you think me handsome? «-Szene abends im Stuhl. Die Besetzung Fassbenders entfernt sich, was seine Statur angeht, zwar ebenfalls von den Beschreibungen des Romantextes. Er ist von sehr schmaler Gestalt, packt jedoch bei der Gartenarbeit selbst mit an. Andererseits kommt Fassbenders Gesicht, kommen seine breite Stirn, die kräftige Nase mit den markanten Nasenflügeln und sein kräftiges Kinn dem Katalog von Rochesters facialen Eigenschaften, den die Ich-Erzählerin entwirft, in hohem Maße entgegen. Ausgreifende Bewegungen fehlen, die häusliche Umgebung scheint ihn eher zu bedrücken, als dass sie - trotz der warmen Farbtöne, die den Raum in einem Licht wie bei Georges de la Tour erscheinen lassen zu seinem Wohlbefinden beitragen würde. Gestisch sich zurücknehmend, kann seine Rede schneidend kurz sein. Es ist Rochesters und Janes zweites Gespräch am Kamin, der szenische Aufbau ist zweigeteilt, Adèle, Sophie und Mrs. Fairfax sind zu Beginn ebenfalls anwesend, verlassen dann aber den Raum, so dass für den Schlagabtausch eine ähnliche Intimität hergestellt wird, wie in der BBC-Version.

Die visuelle Auflösung geht hier jedoch einen anderen Weg: Rochester ist als sitzende Figur zunächst Halbnah/Amerikanisch kadriert, im Bild rechts von ihm der Kamin, dessen Feuer die ihm nähere Gesichtshälfte etwas mehr erhellt. Sein Gesichtsausdruck bewahrt noch den Missmut, den die Erin-

49 Beide Zitate sind der Flashback-Erzählung über die Herkunft Adèles entnommen: Céline betrügt Rochester im Pariser Hotelzimmer mit einem schmucken jungen Offizier, und Rochester wird Zeuge ihrer Intimitäten. 


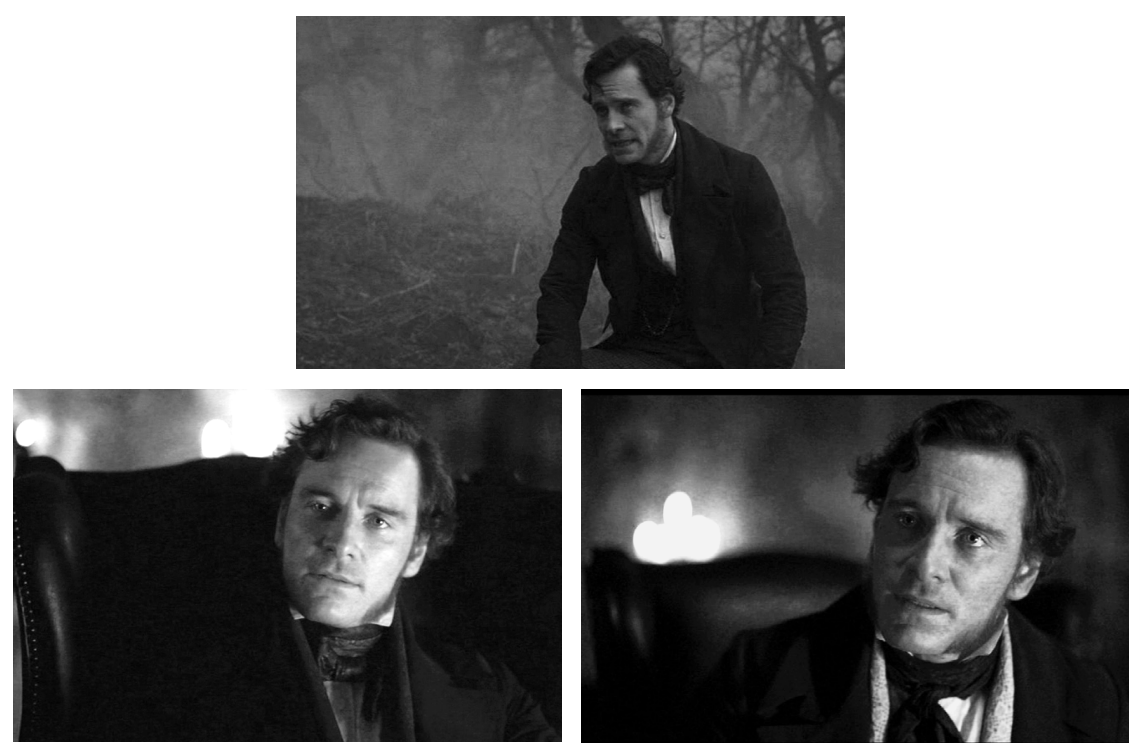

Abb. 16-18: Michael Fassbender als Rochester, 2011

nerung an seine frühere Geliebte Céline Varens in ihm wachgerufen hat, als Adèle sich, es ihrer Mutter gleichtuend, bei ihm für das von der Reise mitgebrachte Kleid bedankt hat. Sie sind nun allein, Jane (Gegenschuss auf Nah) beobachtet ihn ruhig. Ein Anflug von Ekel, oder besser: ein Nachhall davon, grundiert seinen mimischen Ausdruck. Er nippt am Weinglas und blickt zu ihr: "Your gaze is very direct, Miss Eyre. [Er neigt den Kopf etwas und hebt die Hände] Do you think me handsome?« Umschnitt auf Jane (Halbnah) "No, Sir." Umschnitt auf Rochester (Nah/Groß, kleine Pause) "What fault do you find with me? I have all my limbs and features." Umschnitt auf Jane (Nah) »I beg your pardon, Sir. I ought to have replied that beauty is of little consequence. "Umschnitt auf Rochester (Nah), er hält den Blick unverändert. Umschnitt auf Jane (Nah), sie schluckt. Umschnitt auf Rochester (Nah) "You're blushing, Miss Eyre.« Umschnitt auf Jane (Nah) (off) »And though you're not pretty any more than I am handsome, I must say it becomes you."

Janes wie auch Rochesters mimische Reaktionen sind von großer Selbstbeherrschung dominiert, sie halten den Blick ebenso wie sie dem Blick des anderen standhalten. Janes Schlucken deutet an, dass ihre waghalsige, ehrliche Antwort ein großes Risiko bedeutet. Ihr Arbeitgeber lacht diese Antwort aber nicht weg, sondern nimmt sie ernst, verwickelt sie in eine 
Haltung, die auf - genderdifferente - Gleichheit in Bezug auf den Mangel an äußerer Schönheit setzt. Die Augen bleiben fixiert auf sie, nur der Mund bewegt sich. Als sie auf sein Kompliment hin die Augen niederschlägt, löst er die fixierende Haltung auf. Fassbenders Mimik (beim Sprechen bleibt seine Augenpartie konstant unverändert) lässt Rochester mal verhalten aggressiv, mal verstört angesichts ihrer Antworten wirken.

Die Analyse konnte hier nur ansatzweise die Wirkungen herausarbeiten, welche die unterschiedlichen Physiognomien der drei Darsteller auf die Gestaltung der Figur ausüben. Sie sollte immerhin gezeigt haben, dass Besetzungsentscheidungen eng mit Konzepten und jeweils bestimmte Aspekte exponierenden Sichtweisen auf die Figur verbunden sind, die einerseits das Drehbuch an den Roman und andererseits wiederum die Regie und die Schauspieler an das Skript interpretierend anlegen. Hurt und Fassbender agieren mimisch höchst zurückhaltend; umso bedrohlicher für Jane wirkt Rochesters Verhalten bei Fukunaga in der verhinderten Hochzeitsnacht: Sein Besitzanspruch bricht sich aggressiv Bahn, als er ansetzt, ihr die Kehle zuzudrücken, um sie davon abzuhalten, ihn zu verlassen. Stephens spielt Rochesters Emotionen mimisch vielfältiger aus. Sein Spiel unterstreicht, dass dessen Verletzlichkeit, Leidenschaft und Humor jederzeit ins Melancholische und Bittere driften können. Er fordert Jane als seinen Spiegel und ist darin seinerseits ihr ideales Projektionsobjekt.

Aus der vergleichenden Betrachtung der Szenen, ihrem dramaturgischen Aufbau und ihrer je spezifischen visuellen Auflösung wurde aber auch deutlich, dass das Spektrum des Pathognomischen seine Grenze letztlich auch im physiognomischen Fundament der Gesichter hat - in einem Feld, das im Spiel nicht mehr willkürlich steuer- und beherrschbar ist. Dabei dürfte meines Erachtens weniger die Kopf- und Gesichtsform (vgl. den verblüffenden Unterschied zwischen Hurts Profil und seiner Gesichtsvorderansicht in Abb. 9 und 10) als vielmehr die muskuläre Struktur des Gesichts von Bedeutung sein. Denn wie intim die Kommunikation zwischen Jane und Rochester letztlich ausfallen und wie vertrauenerweckend dessen hochambivalenter Charakter phasenweise filmisch ausgedeutet werden kann, hängt auch von den schauspielerischen Möglichkeiten ab, die Bewegung von Augen- und Mundpartie zu verbinden. In einem Medium der Nähe wie dem Fernsehen erweist sich (im Unterschied zum Kino) denn auch Stephens' mimischer Modus als höchst adäquat. ${ }^{50}$

50 Vgl. hierzu den Kommentar der Regisseurin Susanna White: »That was the hardest thing. To find an actor who looked right, who could carry across the humor, the intelligence, 
Ob diese Effekte bei den drei Adaptionen freilich bereits beim Casting im Vorstellungshorizont der Filmschaffenden gewesen sind, ließe sich nur befriedigend klären, wenn es dazu hinreichend differenzierte, die Besetzung reflektierende Paratexte gäbe.

who could be very dark, who could play depression, who could play lightness against that. And would just be knock out gorgeous." Zit. nach: Jane Eyre, DVD, BBC Worldwide 2006, Disc 2, Extras/Interviews, TC 03:20-03:41. 
